- Additional tables are published online only. To view these files please visit the journal online (http://dx.doi.org/ 10.1136/sextrans-2011050406)

${ }^{1}$ Queen Mary University of London, Blizard Institute, Barts Sexual Health Centre, St Bartholomew's Hospital, London, UK

${ }^{2}$ Research Department of Infection and Population Health, University College London, London, UK

${ }^{3}$ Brighton and Sussex Medical School, Mayfield House, University of Sussex, Falmer, Brighton, UK

\section{Correspondence to} Dr John M Saunders, Queen Mary University of London, Blizard Institute, Centre for Immunology and Infectious Disease: Sexual Health \& HIV, Barts Sexual Health Centre, St Bartholomew's Hospital, London EC1A 7BE, UK: j.saunders@qmul.ac.uk

Accepted 20 March 2012

Published Online First 17 April 2012

\title{
Where do young men want to access STI screening? A stratified random probability sample survey of young men in Great Britain
}

\author{
John M Saunders, ${ }^{1}$ Catherine H Mercer, ${ }^{2}$ Lorna J Sutcliffe, ${ }^{1}$ Graham J Hart, ${ }^{2}$ \\ Jackie Cassell, ${ }^{3}$ Claudia S Estcourt ${ }^{1}$
}

\section{ABSTRACT}

Objectives Rates of sexually transmitted infections (STIS) in UK young people remain high in men and women. However, the National Chlamydia Screening Programme has had limited success in reaching men. The authors explored the acceptability of various medical, recreational and sports venues as settings to access self-collected testing kits for STIs and HIV among men in the general population and those who participate in sport.

Methods A stratified random probability survey of 411 (weighted $n=632$ ) men in Great Britain aged 18-35 years using computer-assisted personal and self-interviews.

Results Young men engaged well with healthcare with $93.5 \%$ registered with, and $75.3 \%$ having seen, a general practitioner in the last year. $28.7 \%$ and $19.8 \%$ had previously screened for STIs and HIV, respectively. Willingness to access self-collected tests for STIs (85.1\%) and HIV (86.9\%) was high. The most acceptable pick-up points for testing kits were general practice $79.9 \%$, GUM $66.8 \%$ and pharmacy $65.4 \%$. There was a low acceptability of sport venues as pick-up points in men as a whole (11.7\%), but this was greater among those who participated in sport (53.9\%).

Conclusions Healthcare settings were the most acceptable places for accessing STI and HIV self-testing kits. Although young men frequently access general practice, currently little STI screening occurs in this setting. There is considerable potential to screen large numbers of men and find high rates of infection through screening in general practice. While non-clinical settings are acceptable to a minority of men, more research is needed to understand how these venues could be used most effectively.

\section{INTRODUCTION}

Over the past decade, there has been a sustained rise in the rates of most sexually transmitted infections (STIs) in the UK. ${ }^{1}$ The burden of infection with Chlamydia trachomatis, the most prevalent STI, is shared equally by men and women. ${ }^{2} 3$ Over this time, highly sensitive and specific tests have been developed for the diagnosis of chlamydia and other infections such as Neisseria gonorrhoeae and HIV, which can be performed on non-invasive self-collected samples. ${ }^{4} 5$ As a result, testing for STIs and HIV can now be conducted in a variety of non-healthcare settings without the need for access to microscopy or interaction with healthcare professionals. These technological advances have underpinned development of the National Chlamydia Screening Programme (NCSP) in England and Wales. However, the NCSP has had difficulty screening the required numbers of people needed in order to have the predicted impact on health outcomes. 67

The NCSP tests only half the number of men as women, and the places in which men are tested differ from women. ${ }^{8}$ Of the NCSP tests done in women, more than $50 \%$ are carried out in 'core services' (general practice, contraceptive and sexual health services and pharmacies). ${ }^{7}$ In contrast, around $25 \%$ of tests in men are in 'core services', with testing more commonly occurring in 'outreach' and non-healthcare settings. ${ }^{7}$ Positivity rates of men tested by the NCSP in non-healthcare settings are generally lower than those from men tested in core services. ${ }^{9}$ This highlights the limited impact that screening men in non-healthcare settings may have on public health, as it is the coverage of screening which is important and ensuring that populations with the highest prevalence of infection are tested. ${ }^{6}$ A greater and faster reduction in chlamydia prevalence may be achieved through including more men in screening; however, the cost-effectiveness of screening more men has been recently questioned. ${ }^{10} 11$ Despite this, it is generally accepted that efforts should be made to engage more men with STI screening, and the UK Department of Health has commissioned research to look specifically at this issue. ${ }^{12}$

STI screening in some sports settings has been undertaken in the UK and overseas, in a variety of ways and with varying degrees of success, in an attempt to encourage more men to test for STIs and to engage in general healthcare. ${ }^{13-15}$ In England, after swimming and cycling, more men aged 16 years and over play football (soccer) at least once a month than any other sport and do so in a club infrastructure which could provide support for screening initiatives. ${ }^{16} 17$ Although this suggests that football venues could provide feasible settings in which to provide large numbers of men access to STI and HIV testing, the acceptability of this approach is poorly understood.

Here, we report findings from a stratified random probability survey which explored the medical, social and sporting venues in which men aged 18-35 years and resident in Great Britain find it bmi.com/site/about/unlocked. xhtml 
acceptable to access self-collected testing kits for STIs and HIV. We also aimed to determine whether those men who play football would find their football venues acceptable as pick-up points for self-testing kits in an attempt to further understand the acceptability and feasibility of using football settings to engage men in STI testing.

\section{METHODS}

Our study formed part of the National Centre for Social Research (NatCen) quarterly social research survey (Omnibus). ${ }^{18}$ This is a stratified random probability survey of adults aged over 16 years in Great Britain. A multistage sampling design technique is used for the Omnibus. First, postcode sectors are ordered according to Government Office Regions and the National Statistics Socio-economic Classification before selecting 153 sectors. Twenty addresses are then selected from the Postcode Address File for each of the 153 postcode sectors. This gives a total sample size of 3060 addresses. Finally, a single participant aged over 16 years is selected at random from these addresses, but questions relating to our study were only delivered to men aged between 18 and 35 years. Appropriate selection and calibration weights are applied to correct for the unequal probability of selection in households of different numbers of occupants and to ensure the weighted distributions match population totals. The Omnibus is conducted in accordance with the Social Research Association Ethical guidelines. ${ }^{19}$

We developed 10 questions exploring use of general and sexual healthcare, key sexual risk behaviours, participation in sporting activities and the acceptability of self-collected STI and HIV testing in a variety of medical, social and recreational settings. Where applicable, we used questions validated for the National Surveys of Sexual Attitudes and Lifestyles (Natsal). ${ }^{20}$ Surveys were delivered using a combination of computer-assisted personal and computer-assisted self-interviewing techniques. Questions were piloted with researchers at NatCen prior to inclusion in the survey, and the survey programme was tested for correct routing, and internal range and consistency error warnings were created.

Data were collected in three waves between January and October 2010. Selected participants were sent an invitation letter prior to the interview together with an unconditional $£ 5$ voucher. Interviewers called at each address on at least six, and a maximum of nine, separate occasions at different times of the day and week, including evenings and weekends, before an address was recorded as a non-response. The first three calls were conducted after 18:00 on Monday to Thursday or at a weekend. In an attempt to increase participation and the accuracy of the data recorded, more sensitive questions were completed by participants without the interviewers seeing responses and data immediately and confidentially stored on the laptop computers. Interviews lasted between 25 and $30 \mathrm{~min}$.

Coding of the data was performed by researchers at NatCen. Free-text responses to questions were back-coded where possible into existing codes for that question. New code frames were created for open questions from responses given in initial interviews.

\section{Statistical methods}

A sample size of 225 men aged 18-35 years was calculated to provide adequate statistical power ( $80 \%$ ) to detect clinically important differences in key predictors at the $5 \%$ level. However, over the course of three Omnibus waves, data were collected from 411 men, enabling more precise estimates to be obtained.
The $\chi^{2}$ statistic was used to detect statistically significant differences in proportions between men aged 18-24 years, men aged 25-29 years and men aged 30-35 years. Data were analysed using the statistical package STATA to account for the complex survey design of the Omnibus survey. Statistical significance is considered as $\mathrm{p}<0.05$ for all analyses.

\section{RESULTS}

The three waves of the survey had an overall response rate of $53 \%$. The median age of men was 28 years, with 130 men (38.9\%) aged $18-24$ years, 124 men $(28.2 \%)$ aged $25-29$ years and 157 men (32.9\%) aged $30-35$ years.

\section{Healthcare use and previous STI/HIV testing}

Almost all (93.5\%) men were registered with a general practice and $75.3 \%$ had seen their general practitioner (GP) within the last year (table 1), with no difference by age group. Of men, $28.7 \%$ and $19.8 \%$ had previously tested for STIs and HIV, respectively. Among those who had tested for STIs, 68.2\% (95\% CI $52.2 \%$ to $80.7 \%$ ) of men $<25$ years of age had done so in the last year compared with $30.4 \%$ (95\% CI $17.1 \%$ to $48.1 \%$ ) of men aged $25-29$ years and $9.1 \%$ (95\% CI $2.3 \%$ to $29.8 \%$ ) of men aged $30-35$ years $(p<0.001)$. Of the men who had previously tested for HIV, those under 25 years were more likely to have tested in the last year than older men, $69.9 \%$ (95\% CI $45.7 \%$ to $86.5 \%$ ) and $20.0 \%$ ( $95 \%$ CI $10.4 \%$ to $35.0 \%$ ), respectively ( $p=0.0004$ ). The majority of STI and HIV tests had been performed in a clinical setting with over half reporting testing in sexual health (genitourinary medicine) clinics, while approximately one in six had tested in general practice. Relatively few men reported testing for STIs in non-clinical settings.

\section{Behavioural factors}

Of all men, $86.2 \%$ reported at least one sexual partner in the last year with $73.4 \%$ reporting at least one sexual partner over the last 3 months (table 2). Younger men reported greater numbers of sexual partners over the last year and the last 3 months compared with men in older age groups $(p<0.001$ and $p=0.003$, respectively). Condom use was greatest in men under 25 years with $34.7 \%$ reporting using condoms every time they had sex in the last 3 months in comparison to less than one-quarter of men aged 25 years and older $(p<0.001)$. Among men who had had sex, $3.8 \%$ reported that the gender of their last sexual partner was male and this did not vary by age group.

\section{Willingness to use self-collected testing kits for STIs (urine) and HIV (oral fluid) and acceptability of different settings}

The majority of men were willing to provide a self-collected sample for STI/HIV testing (table 3). Specifically, 85.1\% reported that they were willing to provide a urine sample for STI testing with no variation by age group, while $86.9 \%$ of all men reported their willingness to provide an oral fluid sample for HIV testing, although this did vary by age group from $79.7 \%$ of men aged $25-29$ years to $95.0 \%$ of men aged $18-24$ years $(p=0.001)$. General practice surgeries $(79.7 \%)$, sexual health clinics $(66.8 \%)$ and pharmacies $(65.4 \%)$ were the most acceptable test kit pickup points with no variation by age. Further education settings were more popular than school settings as pick-up points $(41.6 \%$ vs $28.1 \%$ ), while the workplace was acceptable to $22.4 \%$ of all men. Gym and sports centres were considered acceptable pickup points by $18.5 \%$ and $13.4 \%$, respectively, of all men, with no variation by age. 
Table 1 Factors related to health service use among men aged $18-35$ years

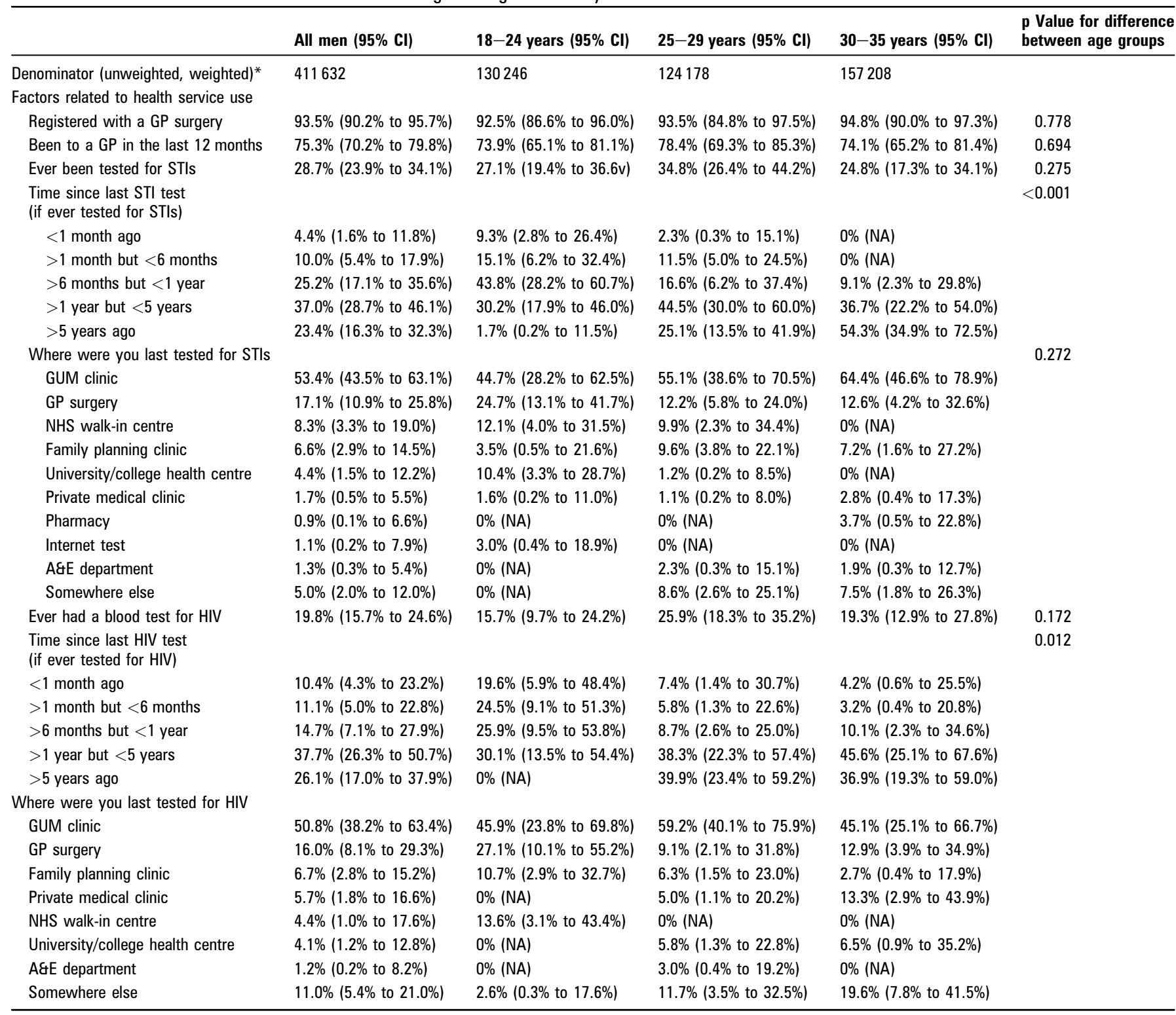

*Percentages presented for weighted sample.

GP, general practice; NA, not applicable; STIs, sexually transmitted infections.

\section{Participation in sport}

Of all men, $69.4 \%$ (95\% CI $63.9 \%$ to $74.5 \%$ ) had participated in a sporting activity at least once within the 4 weeks prior to interview with this proportion greatest among men aged over 30 years $(78.3 \%$; $95 \%$ CI $69.7 \%$ to $84.9 \%$ ) than among those aged $18-29$ years $(65.9 \%$; $95 \%$ CI $59.0 \%$ to $72.2 \%)(p=0.0189)$ (data available as online supplement). Among all men aged 18-35 years, the five most popular activities to participate in were football (soccer) (52.9\%; 95\% CI $46.2 \%$ to 59.5\%), jogging (45.4\%; $95 \%$ CI $38.7 \%$ to $52.2 \%$ ), gym (36.8\%; $95 \%$ CI $30.3 \%$ to $43.8 \%)$, cycling (31.9\%; $95 \%$ CI $26.0 \%$ to $38.6 \%)$ and swimming $(29.6 \%$; $95 \%$ CI $23.9 \%$ to $35.9 \%)$. Men under 30 years were more likely to have participated in football than those aged at least 30 years $(57.5 \%$; $95 \%$ CI $49.2 \%$ to $65.4 \%$ ) versus $43.3 \%$ (95\% CI $33.1 \%$ to $54.0 \%, \mathrm{p}=0.033$ ), with $74.4 \%$ ( $95 \%$ CI $65.2 \%$ to $81.8 \%)$ of men who played football reporting to play at least once a week (no variation by age group).

As reported above, there was generally low acceptability of sports settings as pick-up points for STI and HIV testing kits but, among those who reported participation in a sporting activity within the last 4 weeks, $53.9 \%$ (95\% CI $46.9 \%$ to $60.8 \%$ ) and $51.6 \%$ (95\% CI $44.8 \%$ to $58.4 \%$ ) said they would be willing to pick-up STI and HIV testing kits from the place of activity, respectively, with no significant variation by age group. Among the 129 men who reported playing football in the last 4 weeks, these figures were $47.3 \%$ ( $95 \%$ CI $37.2 \%$ to $57.6 \%$ ) and $43.5 \%$ ( $95 \%$ CI $34.1 \%$ to $53.3 \%$ ), respectively. There was no difference in healthcare use between men who had and had not played football in the previous 4 weeks with equal proportions being registered with a GP, having seen a GP in the last year and having undergone previous testing for STIs and HIV. Men who played football reported similar numbers of sex partners and were as likely to report their last sex partner as being male as men who did not play football (data available as online supplement).

\section{DISCUSSION}

Overall, men appeared well engaged with healthcare with almost all being registered with a GP and three-quarters having 
Table 2 Key sexual risk behaviours among men aged 18-35 years

\begin{tabular}{|c|c|c|c|c|c|}
\hline & All men $(95 \%$ Cl) & $18-24$ years $(95 \% \mathrm{CI})$ & $25-29$ years $(95 \% \mathrm{Cl})$ & $30-35$ years $(95 \% \mathrm{Cl})$ & $\begin{array}{l}\text { p Value for difference } \\
\text { between age groups }\end{array}$ \\
\hline Denominator (unweighted, weighted) ${ }^{*}$ & 411632 & 130246 & 124178 & 157208 & \\
\hline \multicolumn{6}{|l|}{ Behavioural factors: } \\
\hline 0 & $13.8 \%(10.1 \%$ to $18.7 \%)$ & $17.8 \%(11.1 \%$ to $27.4 \%)$ & $13.8 \%(7.8 \%$ to $23.2 \%)$ & $8.2 \%(4.5 \%$ to $14.6 \%)$ & \\
\hline 1 & $61.4 \%(55.8 \%$ to $66.6 \%)$ & $43.5 \%(34.6 \%$ to $52.9 \%)$ & $66.2 \%$ (56.2\% to $74.9 \%)$ & $81.8 \%(73.7 \%$ to $87.8 \%)$ & \\
\hline 2 & $8.9 \%(6.2 \%$ to $12.5 \%)$ & $14.0 \%(8.8 \%$ to $21.5 \%)$ & $6.0 \%(2.8 \%$ to $12.6 \%)$ & $4.5 \%(2.3 \%$ to $8.6 \%)$ & \\
\hline $\begin{array}{l}\text { Median number of partners } \\
\text { (lower, upper quartiles) }\end{array}$ & $1(1,2)$ & $1(1,2)$ & $1(1,1)$ & $1(1,1)$ & \\
\hline Number of partners in the last 3 months & & & & & 0.003 \\
\hline 0 & $26.6 \%(21.4 \%$ to $32.6 \%)$ & $34.4 \%(25.3 \%$ to $44.9 \%)$ & $26.7 \%(18.1 \%$ to $37.4 \%)$ & $15.4 \%(10.1 \%$ to $23.0 \%)$ & \\
\hline 1 & $65.4 \%(59.3 \%$ to $70.9 \%)$ & $52.7 \%(42.5 \%$ to $62.6 \%)$ & $66.9 \%$ (55.8\% to $76.4 \%)$ & $81.7 \%(73.6 \%$ to $87.7 \%)$ & \\
\hline $2+$ & $8.1 \%(5.4 \%$ to $11.9 \%)$ & $12.9 \%(7.6 \%$ to $21.1 \%)$ & $6.4 \%(3.1 \%$ to $12.9 \%)$ & $2.9 \%(0.9 \%$ to $9.0 \%)$ & \\
\hline $\begin{array}{l}\text { Last sexual partner was male } \\
\text { (if had sex) }\end{array}$ & $3.8 \%(2.2 \%$ to $6.4 \%)$ & $4.5 \%(1.9 \%$ to $10.5 \%)$ & $4.9 \%(2.1 \%$ to $11.2 \%)$ & $1.6 \%(0.5 \%$ to $5.4 \%)$ & 0.345 \\
\hline
\end{tabular}

*Percentages presented for weighted sample.

seen their GP in the last year. Awareness of sexual health appeared high as almost a third of men reported screening for STIs and a fifth reported testing for HIV. The most acceptable venues for young men to pick-up self-collected STI and HIV test kits were healthcare settings (general practice, sexual health clinics, pharmacies), whereas sport, social and recreational venues were acceptable to a smaller proportion of men. Football (soccer) was the most popular sport and around half of men who played football reported that they would find their football venue an acceptable place to access STI and HIV testing kits.

To our knowledge, this is the first stratified probability survey of young men in Britain to determine the acceptability of various settings for accessing self-collected STI and HIV test kits. It provides generalisable data which should benefit those involved in researching, developing and delivering STI services for men in healthcare and non-healthcare settings within Britain, particularly in the context of low uptake of testing in men reported by the NCSP. However, we do not know the reasons for refusal to take part in the survey or how those who declined differ from participants, although we did weight the sample in an attempt to adjust for a lower response among younger men. Differences in acceptability between ethnic groups and whether respondents live in urban or rural areas are unknown as our sample was not powered to look for these

Table 3 Willingness to use self-collected sexually transmitted infection (STI) and HIV testing kits and acceptable pick-up points for tests among men aged $18-35$ years

\begin{tabular}{|c|c|c|c|c|c|}
\hline Factors & All men (95\% Cl) & $18-24$ years $(95 \% \mathrm{Cl})$ & $25-29$ years $(95 \% \mathrm{Cl})$ & $30-35$ years $(95 \% \mathrm{Cl})$ & $\begin{array}{l}\text { p Value for difference } \\
\text { between age groups }\end{array}$ \\
\hline Denominator (unweighted, weighted) ${ }^{*}$ & 411632 & 130246 & 124178 & 157208 & \\
\hline \multicolumn{6}{|c|}{ Willingness to use self-collected testing kits for STI/HIV testing } \\
\hline $\begin{array}{l}\text { Willing to provide mouth swab for } \\
\text { HIV testing }\end{array}$ & $86.9 \%(82.4 \%$ to $90.4 \%)$ & $95.0 \%(89.7 \%$ to $97.6 \%)$ & $79.7 \%(69.9 \%$ to $87.0 \%)$ & $82.7 \%(74.2 \%$ to $88.9 \%)$ & 0.001 \\
\hline \multicolumn{6}{|c|}{ Acceptable pick-up points for testing kits } \\
\hline GUM clinic & $66.8 \%(60.8 \%$ to $72.3 \%)$ & & & & \\
\hline Pharmacy & $65.4 \%(59.4 \%$ to $71.0 \%)$ & & & & \\
\hline Sent in the post & $52.2 \%(46.4 \%$ to $58.0 \%)$ & & & & \\
\hline College/university campus & $41.6 \%(35.9 \%$ to $47.6 \%)$ & & & & \\
\hline School & $28.1 \%(23.0 \%$ to $33.8 \%)$ & & & & \\
\hline Workplace & $22.4 \%(17.8 \%$ to $27.8 \%)$ & & & & \\
\hline Sports club & $11.7 \%(8.4 \%$ to $16.1 \%)$ & & & & \\
\hline Coffee shop/cafe & $6.9 \%(4.6 \%$ to $10.3 \%)$ & & & & \\
\hline Other & $0.4 \%(0.0 \%$ to $3.0 \%)$ & & & & \\
\hline
\end{tabular}

*Percentages presented for weighted sample. 
associations. Although the questions we developed did not undergo formal psychometric testing, where possible we used questions that had been validated for use in the highly regarded Natsal surveys. ${ }^{20}$

While many studies have shown that using non-traditional and sport settings to screen for STIs is feasible, ${ }^{13}{ }^{21}$ few have focused on the acceptability of different settings for men. Lorimer's study of willingness to participate in a non-medical approach to chlamydia screening found that men in particular valued the possibility of screening in these settings. ${ }^{22}$ Furthermore, the uptake of screening varied by setting, supporting our finding that acceptability of sport settings was greater in those who had actually engaged in sporting activities over the last month. ${ }^{15}$ Anonymity appeared to be a key factor determining acceptability of screening in a qualitative study of young men's experiences and perceptions of chlamydia screening commissioned by the NCSP. ${ }^{23}$ Men in that study rejected many of the proposed sport and social venues for fear of the stigma of being seen to take a test. Men also appeared to perceive a degree of incongruity between attending these locations for recreation and the health message of screening. ${ }^{23}$

Our finding that most men had seen their GP in the last year is in keeping with other studies and taken together with the high acceptability of general practice for accessing STI, and HIV testing kits highlights the importance of, and potential for, chlamydia and HIV screening in general practice, especially among younger men. ${ }^{9}{ }^{24}$ However, current rates of STI and HIV screening in general practice are low, which could suggest reluctance on the part of the healthcare provider to offer testing. ${ }^{25}$ Many barriers exist to both opportunistic chlamydia screening and HIV testing in general practice. ${ }^{26-29}$ A move towards opt-out chlamydia testing in general practice could potentially lead to a significant rise in the numbers of tests performed as seen with opt-out HIV testing in general practice and other settings. ${ }^{30} 31$ However, the cost-effectiveness of this approach would need to be examined as prioritising chlamydia screening in general practice may require financial incentives through Quality and Outcomes Framework targets. ${ }^{32}$

A substantial proportion of men in our study reported previously testing for STIs (28.7\%) and HIV (19.8\%) mostly within specialist sexual health settings. Among 18-24-year-olds, over one-quarter reported that they had previously been tested for STIs; however, the NCSP data suggest a coverage of around $12 \%$ of 16-24-year-olds in England and Wales. ${ }^{8}$ This probably reflects testing in GUM services among participants in our survey. However, the reasons for seeking a previous test are not known and may reflect the fact that men had symptoms at that time. The use of self-collected urine testing kits would only be appropriate for asymptomatic men and would also miss infections at non-genital sites. This may be particularly important for men who have sex with men, although self-collected rectal and pharyngeal swabs perform as well as provider-taken swabs and seem to be acceptable, although their use in general practice has not been evaluated to our knowledge. ${ }^{33-35}$ Self-collected specimens for syphilis testing is feasible using dried blood from a finger prick and appears to be acceptable among men who have sex with men recruited from non-clinical settings in one study. ${ }^{36} 37$

Our data reveal a testing rate for HIV which is higher than equivalent data from Britain's most recent Natsal, which found that $6.63 \%(95 \%$ CI $5.14 \%$ to $8.52 \%)$ of men aged $16-24$ years and $14.8 \%$ (95\% CI $13.0 \%$ to $16.7 \%$ ) of men aged $25-34$ years reported having had a blood test for HIV. ${ }^{26}$ Both our data and the Natsal data excluded blood donation as a reason for an HIV test, although the Natsal data are now a decade old.

\section{Key messages}

The burden of STI in the UK is similar in men and women but men are perceived as hard to reach for STI screening.

- Three-quarters of men have seen their GP in the last year but relatively little screening occurs in general practice.

- Young men are willing to test for STIs and primary care settings are the most acceptable locations.

- General practice has considerable potential to widen access to STI and HIV screening for young men.

It is important that venues in which STI screening and testing kits are offered are acceptable to target populations. While it is now possible to deliver testing in non-clinical settings, this research highlights that, among 18-35-year-old men in Britain at least, it is the traditional healthcare settings that are most acceptable as pick-up points for self-testing kits. Young men frequently access primary care, and we feel that there is considerable potential to engage more men in STI and HIV testing through general practice. ${ }^{38}$ The NCSP data shows that only $25 \%$ of tests in men are done in 'core' health settings. ${ }^{8}$ In contrast, our research shows that these are the preferred access points for as many as $80 \%$ of men. Based on our findings, there is clearly a mismatch between where services are currently provided and the settings in which men prefer to access STI and HIV screening. While non-traditional settings are acceptable to a minority of men and may be important in reaching men who would otherwise not seek STI screening, given the high levels of acceptability of traditional services, further research into outreach screening must include appropriate analysis of the costeffectiveness and public health impact so that resources are used most appropriately. This is especially important when considering the potentially low rates of chlamydia detected among the men screened in non-healthcare settings. ${ }^{9}$ While sport and other non-healthcare settings are acceptable pick-up points for some men, more research is needed to understand how these venues could be used most effectively for public health.

Acknowledgements The authors thank National Centre for Social Research (NatCen).

Contributors JC had the original idea for the paper. CSE was the principal investigator of the study and obtained funding from the National Institute for Health Research. JMS and LJS developed the questions for the survey. JMS wrote the first draft of the paper and coordinated the writing of the paper, with all authors contributing to subsequent drafts. Plans for analysis were led by CHM who undertook all statistical analyses. CSE is the paper's guarantor.

Funding This article represents independent research commissioned by the National Institute for Health Research (NIHR) under its Programme Grants for Applied Research scheme (RP-PG-0707-10208). The views expressed in this article are those of the authors and not necessarily those of the NHS, NIHR or the Department of Health.

\section{Competing interests None.}

Provenance and peer review Not commissioned; externally peer reviewed.

\section{REFERENCES}

1. Health Protection Agency. Number of selected STI diagnoses made at genitourinary medicine clinics in the UK and England: 2000-2009. 2000-2009. http://www.hpa.org.uk/web/HPAFile/HPAweb C/1215589013442

2. Macleod J, Salisbury C, Low N, et al. Coverage and uptake of systematic postal screening for genital Chlamydia trachomatis and prevalence of infection in the United Kingdom general population: cross sectional study. BMJ 2005;330:940.

3. Fenton KA, Korovessis C, Johnson AM, et al. Sexual behaviour in Britain: reported sexually transmitted infections and prevalent genital Chlamydia trachomatis infection. Lancet 2001;358:1851-4.

4. British HIV Association. UK National Guidelines for HIV Testing 2008. 2008. http:// www.bhiva.org/documents/Guidelines/Testing/GlinesHIVTest08.pdf 
5. Ross JC, Ison CA, Carder C, et al. Sexually Transmitted Infections: UK National Screening and Testing Guidelines. British Association for Sexual Health and HIV, 2006. http://www.bashh.org/documents/59/59.pdf

6. Turner KM, Adams EJ, Lamontagne DS, et al. Modelling the effectiveness of chlamydia screening in England. Sex Transm Infect 2006;82:496-502.

7. National Chlamydia Screening Programme. England Quarters 1-3 April December 2010. 2011. http://www.chlamydiascreening.nhs.uk/ps/assets/pdfs/data/ sha_presentations11/01-3_2010_National.pdf

8. National Chlamydia Screening Programme. Data for the Period 1st April 2010-31st Dec 2010. NCSP and non-GUM, non-NCSP tests based on VSI criteria. 2011. http://www.chlamydiascreening.nhs.uk/ps/assets/pdfs/data/ PCT Detailed Tables-Apr10-Dec10.pdf

9. Johnson SA, Simms I, Sheringham J, et al. The implementation of chlamydia screening: a cross-sectional study in the south east of England. Sex Transm Infect 2010;86:217-21.

10. Kufeji 0, Slack R, Cassell JA, et al. Who is being tested for genital chlamydia in primary care? Sex Transm Infect 2003;79:234-6.

11. Turner K, Adams E, Grant A, et al. Costs and cost effectiveness of different strategies for chlamydia screening and partner notification: an economic and mathematical modelling study. BMJ 2011;342:c7250.

12. Forrest S, Lloyd T. Engaging Young Men in the National Chlamydia Screening Programme: Some Recommendations for the Implementation of the 'Men Too' Strategy. 2011. http://www.boysdevelopmentproject.org.uk/downloads/reports/ WWM_chlamydia_briefing 11.pdf

13. Kong $\overline{\mathbf{F Y}}$, Hocking JS, Link CK, et al. Sex and sport: chlamydia screening in rural sporting clubs. BMC Infect Dis 2009;9:73.

14. Powell J, O'Connor $\mathrm{C}, \mathrm{O}^{\prime} \mathrm{Hlarlaithe} \mathrm{M}$, et al. Chlamydia trachomatis prevalence in men in the mid-west of Ireland. Sex Transm Infect 2004:80:349-53.

15. Lorimer K, Reid ME, Hart GJ. Willingness of young men and women to be tested for Chlamydia trachomatis in three non-medical settings in Glasgow, UK. J Fam Plann Reprod Health Care 2009;35:21-6.

16. Sport England. Once A Month Participation Rates by Sport: Results From JAN 2010-JAN 2011. Sport England, 2011. http://www.sportengland.org/research/ active people survey/idoc.ashx?docid=3e205383-c872-4b63-aff3cebc72f0c1da'sversion=1 (accessed 6 Apr 2012).

17. Sport England. Club Membership by Sport: Results From Jan 2010-Jan 2011. Sport England, 2011. http://www.sportengland.org/research/active_people_survey/ idoc. ashx?docid=4e3a8ed7-7232-4919-8514-147b22a11d91\&version=1 (accessed 6 Apr 2012).

18. National Centre for Social Research. Omnibus. http://www.natcen.ac.uk/study/ omnibus

19. Social Research Association. Ethical Guidelines. 2003. http://www.the-sra.org uk/documents/pdfs/ethics03.pdf

20. National Centre for Social Research. National Surveys of Sexual Attitudes and Lifestyles. 2011. http://www.natcen.ac.uk/study/natsal

21. Ford CA, Viadro Cl, Miller WC. Testing for chlamydial and gonorrheal infections outside of clinic settings: a summary of the literature. Sex Transm Dis 2004;31:38-51.
22. Lorimer K, Reid ME, Hart GJ. "It has to speak to people's everyday life.": qualitative study of men and women's willingness to participate in a non-medical approach to Chlamydia trachomatis screening. Sex Transm Infect 2009;85:201-5.

23. National Chlamydia Screening Programme. Young Men Chlamydia Screening Programme. A Qualitative Evaluation Amongst Young Men. 2009. http://www. chlamydiascreening.nhs.uk/ps/implementation/engaging.html

24. Salisbury C, Macleod J, Egger M, et al. Opportunistic and systematic screening for chlamydia: a study of consultations by young adults in general practice. $\mathrm{Br} \mathrm{J}$ Gen Pract 2006:56:99-103.

25. Sadler KE, Low N, Mercer $\mathrm{CH}$, et al. Testing for sexually transmitted infections in general practice: cross-sectional study. BMC Public Health 2010;10:667.

26. McNulty CA, Freeman E, Bowen J, et al. Barriers to opportunistic chlamydia testing in primary care. Br J Gen Pract 2004;54:508-14.

27. McNulty CA, Freeman E, Bowen J, et al. Diagnosis of genital chlamydia in primary care: an explanation of reasons for variation in chlamydia testing. Sex Transm Infect 2004;80:207-11.

28. Petlo T, Fairley CK, Whitton B, et al. HIV-testing of men who have sex with men: variable testing rates among clinicians. Int J STD AIDS 2011;22:727-9.

29. Sawleshwarkar S, Harrison C, Britt H, et al. Determinants of HIV testing. Sex Transm Infect 2011:87:426-32.

30. Madge S, Smith C, Evans A, et al. Patterns of HIV testing at a London teaching hospital between 2004 and 2007. Int J STD AIDS 2011:22:730-3.

31. Heijman RLJ, Stolte IG, Thiesbrummel HF, et al. Opting out increases HIV testing in a large sexually transmitted infections outpatient clinic. Sex Transm Infect 2009:85:249-55

32. McNulty CA, Freeman E, Howell-Jones R, et al. Overcoming the barriers to chlamydia screening in general practice-a qualitative study. Fam Pract 2010;27:291-302.

33. van der Helm JJ, Hoebe CJ, van Rooijen MS, et al. High performance and acceptability of self-collected rectal swabs for diagnosis of Chlamydia trachomatis and Neisseria gonorrhoeae in men who have sex with men and women. Sex Transm Dis 2009;36:493-7.

34. Wayal S, Llewellyn C, Smith H, et al. Self-sampling for oropharyngeal and rectal specimens to screen for sexually transmitted infections: acceptability among men who have sex with men. Sex Transm Infect 2009;85:60-4.

35. Alexander S, Ison C, Parry J, et al. Self-taken pharyngeal and rectal swabs are appropriate for the detection of Chlamydia trachomatis and Neisseria gonorrhoeae in asymptomatic men who have sex with men. Sex Transm Infect 2008;84:488-92.

36. Brown BS, Klapper PE, Guiver M. P.049 Development of diagnostic serological and molecular screening from dried blood spots for HCV, HIV, HBV and syphilis. J Clin Virol 2009;44(Suppl 1):S27-8.

37. Lee D, Fairley $C$, Cummings $R$, et al. Men who have sex with men prefer rapid testing for syphilis and may test more frequently using it. Sex Transm Dis 2010:37:557-58

38. Hughes G, Williams T, Simms I, et al. Use of a primary care database to determine trends in genital chlamydia testing, diagnostic episodes and management in UK general practice, 1990-2004. Sex Transm Infect 2007:83:310-13. 